\title{
Analysis of spinocerebellar ataxia types 1, 2, 3, and 6, dentatorubral-pallidoluysian atrophy, and Friedreich's ataxia genes in spinocerebellar ataxia patients in the UK
}

Department of Medical Genetics, Box 158, Addenbrooke's NHS Trust, Hills Road, Cambridge CB2 2QQ, UK

J Leggo

D C Rubinsztein

Centre for Human Genetics, Sheffield Children's Hospital NHS Trust, 117 Manchester Road, Sheffield, UK

A Dalton

Department of Medical Genetics, Belfast City Hospital, Belfast, Northern

Ireland

P J Morrison

Department of Medical Genetics, St Mary's Hospital, Hathersage Road, Manchester, UK

A Dodge

Wessex Regional Genetics Laboratory, Salisbury District Hospital, Salisbury SP2 8BJ, UK

M Connarty

Department of Human Genetics, University of Stellenbosch, PO Box

19063, Tygerberg,

South Africa

M J Kotze

Correspondence to: Dr Rubinsztein.

Received 7 May 1997 Revised version accepted for publication 22 July 1997

Jayne Leggo, Ann Dalton, Patrick J Morrison, Alan Dodge, Margaret Connarty, Maritha J Kotze, David C Rubinsztein

\begin{abstract}
Accurate clinical diagnosis of the spinocerebellar ataxias (SCAs) can be difficult because of overlap in phenotype with other disorders and variation in clinical manifestations. Six SCA loci have been mapped and four disease causing genes identified, in addition to the causative gene for Friedreich's ataxia (FA). All of the identified mutations are expansions of trinucleotide repeat tracts. The SCA2 and SCA6 genes were published recently. The extent of the normal CAG size ranges at these loci and the relative frequencies of the known causes of SCA in the UK are not known. This study first investigated the normal size ranges of the SCA2 and SCA6 loci by genotyping control populations of West African and South African subjects, since African populations generally show the greatest allelic diversity. We found one allele larger than the previously determined normal range for SCA2, and our results at the SCA6 locus agreed with the previously reported normal range. The second component of the study assessed the relative frequencies of the SCA1, 2, 3, and 6, DRPLA, and FA trinucleotide repeat mutations in 146 patients presenting with SCA-like symptoms referred to genetic diagnostic laboratories in the UK. We detected mutations in $14 \%$ of patients referred with a diagnosis of autosomal dominant SCA, and in $15 \%$ of patients referred with spinocerebellar ataxia where we did not have sufficient family history data available to allow categorisation as familial or sporadic cases. Friedreich's ataxia accounted for $3 \%$ of the latter category of cases in our sample, but the most common causes of SCA were SCA2 and SCA6.

$(\mathcal{M}$ Med Genet 1997;34:982-985)
\end{abstract}

Keywords: trinucleotide repeat; spinocerebellar ataxia; Friedreich's ataxia

The hereditary ataxias are a group of neurodegenerative disorders characterised by varying degrees of brain stem and cerebellar pathology or dysfunction. Clinical features usually present in the third or fourth decade and can include dysarthria, dementia, pyramidal signs, and oculomotor and spinal cord dysfunction, in addition to cerebellar manifestations. ${ }^{12}$ The dominantly inherited spinocerebellar ataxias (SCAs) are a phenotypically heterogeneous group of disorders which can be difficult to diagnose. In the past two decades at least six SCA loci have been genetically mapped (SCA1-5, 7) and four disease causing genes have been identified (SCA1, 2, 3, and 6). .9 $^{3-9}$ Friedreich's ataxia (FA) has a phenotype which can overlap with the autosomal dominant SCAs, but differs in that it is inherited in a recessive manner and usually presents at an earlier age. It is a progressive ataxia with onset usually before the age of 25 , which can be accompanied by absence of lower limb reflexes, dysarthria, limb weakness, skeletal deformities, and sometimes cardiomyopathy and diabetes. $^{21011}$ However, a significant proportion of patients with the FA mutation have a later age of onset and incomplete presentation (for example, intact reflexes), suggesting that this disease has a broader clinical presentation. ${ }^{1011}$

SCA1, 2, 3, and 6 are associated with abnormal expansions of CAG repeats in the coding regions of their respective genes. ${ }^{3-9}$ Thus, they share the same mutation as spinobulbar muscular atrophy (SBMA), Huntington's disease (HD), and dentatorubral-pallidoluysian atrophy (DRPLA). ${ }^{12}$ In each of these diseases the tracts of CAG repeats are translated into glutamines. In Friedreich's ataxia, more than $95 \%$ of disease alleles are associated with expansion of a GAA trinucleotide repeat tract in the first intron of the causative gene. ${ }^{13}$ This is a large expansion (200 to $>900$ repeats) which correlates inversely with age of onset of the disease. ${ }^{1011}$

The wide variation in clinical manifestations of the SCAs and the overlap in phenotype with other inherited disorders such as DRPLA, FA, and familial spastic paraplegia can confound accurate clinical diagnosis. Molecular characterisation vastly improves upon the use of clinical and histopathological material alone to classify this heterogeneous group of disorders. Identification of a causative mutation can provide a diagnosis in cases who present with minor signs and symptoms, which can manifest years before appearance of the usual symptoms at disease onset, ${ }^{1}$ and allows for the options of 
predictive testing of asymptomatic at risk subjects and prenatal diagnosis.

Before routine diagnostic testing can be offered, it is critical to know the normal and disease size ranges for these trinucleotide repeat tracts. These size ranges need to be determined using a variety of control populations in order to account for the global variation in allele frequencies among populations. ${ }^{14}$ We and others have looked at normal and disease ranges in HD, MachadoJoseph disease (MJD), and myotonic dystrophy (DM) in various populations and have found that there can indeed be variation in allele size ranges among them. ${ }^{15-18}$

The normal repeat size ranges in the recently identified SCA2 and SCA6 genes were established using Japanese and white populations for $S C A 2^{6-8}$ and a small white sample in the case of SCA $6,{ }^{9}$ which may not reflect the true genetic diversity in the total human population. Furthermore, while virtually all of the other diseases associated with CAG expansions have more than 36 repeats in their disease ranges,
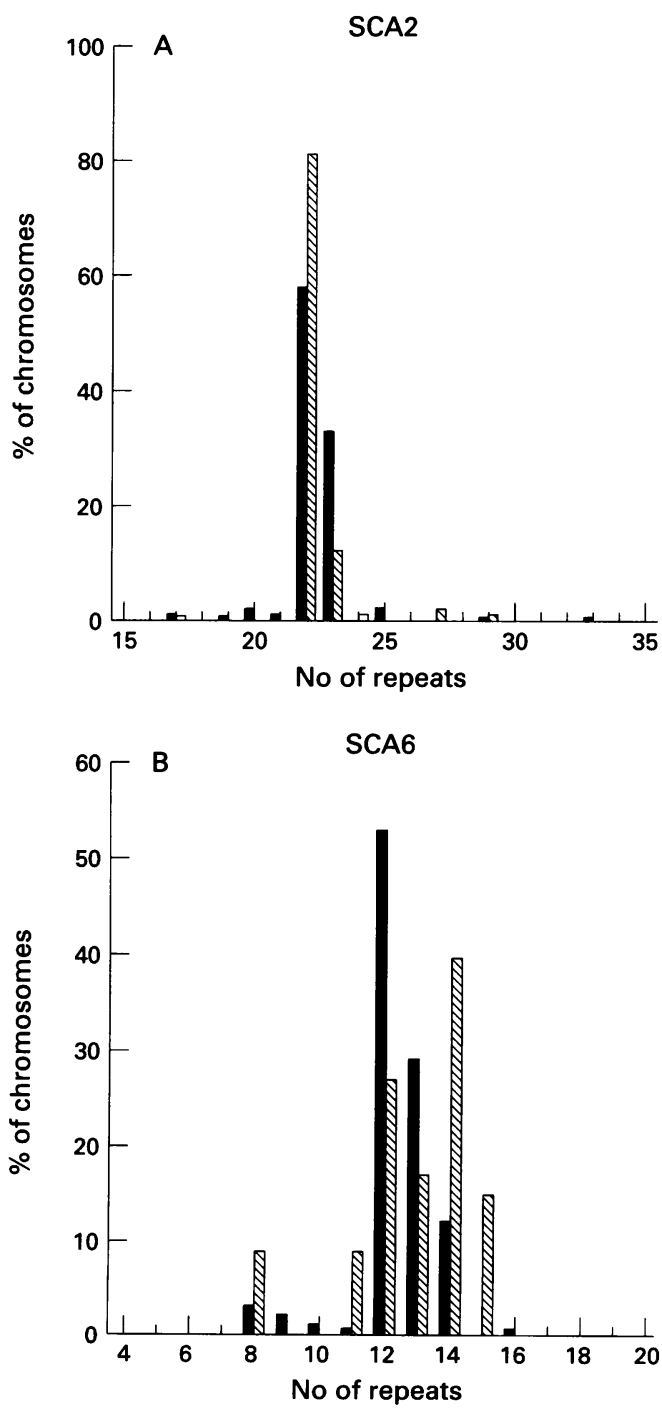

Figure 1 Distributions of numbers of $C A G$ repeats at the $S C A 2(A)$ and SCA6 (B) loci in two African populations (solid bars) and normal UK chromosomes $(n=235)$ (hatched bars). The normal UK chromosomes include data from SCA patients analysed in Cambridge. The distributions indicate the percentage of chromosomes with a given $C A G$ repeat length.
SCA6 seems to be exceptional in that it has a disease range of only 21-27 CAG repeats, ${ }^{9}$ similar in length to many normal chromosomes in related disorders. ${ }^{12}$ To address these issues, we have looked at the allele frequencies of SCA2 and SCA6 in people from Nigeria and South Africa, since African populations are thought to have a high level of allelic diversity at most loci. ${ }^{14}$

In view of the recent identification of SCA2 and SCA6 genes and the lack of knowledge of the relative frequencies of disease causing mutations in the UK, we have determined the relative frequencies of SCA1, 2, 3, and 6 , DRPLA, and FA in a sample of 146 subjects referred for diagnostic testing for ataxia to molecular genetics laboratories in England and Northern Ireland. Knowledge of the relative frequencies of these diseases in the UK will allow realistic expectations of the proportion of patients for whom clinicians can expect positive results.

\section{Methods}

SUBJECTS

Control populations comprised 50 West Africans (mainly from Nigeria) and 100 South African Bantu speaking subjects collected in Cape Town and Johannesburg. All samples were anonymised and apparently unrelated. Although we are not aware that any controls had neurological difficulties, these were not systematically excluded by clinical examination.

The clinical sample comprised 146 patients presenting with SCA-like symptoms referred to genetics diagnostic laboratories in Sheffield (32 males, 34 females, mean age 47 (SD 18), range 2-76 years), Salisbury (nine males, 10 females, mean age 46 (SD 16), range 6-72 years), Manchester (18 males, 18 females, mean age 44 (SD 18), range 3-75 years), Belfast (three males, three females, mean age 42 (SD 11), range 22-49 years) and Cambridge (12 males, seven females, mean age 46 (SD 17), range 28-78 years). This sample included all patients referred to these laboratories with SCA-like symptoms and included cases where a positive result had previously been obtained for SCA1, SCA3, or DRPLA. Twenty-two of these were diagnosed as having autosomal dominant SCA and family histories were complete. The family history details on the laboratory referral forms of 124 cases were not sufficient to allow categorisation as truly sporadic or familial cases and two patients were referred with a diagnosis of familial SCA, but with incomplete family histories. Neurologists referred $98 \%$ of cases from Sheffield, $79 \%$ of cases in Salisbury, $67 \%$ of cases from Belfast, $52 \%$ of cases from Manchester, and $47 \%$ of cases from Cambridge. The other referring clinicians comprised mainly geneticists but included a GP, paediatricians, and psychiatrists. There was no physician who referred the bulk of patients within any one centre.

PCR ANALYSIS

The SCA2 CAG repeat expansion was analysed using conditions and primers SCA2-A 
Table 1 Distribution of mutations at known disease loci in patients presenting with SCA-like symptoms in the UK

\begin{tabular}{|c|c|c|c|}
\hline Locus & $\begin{array}{l}\text { Isolated cases (no } \\
\text { family history) }\end{array}$ & $\begin{array}{l}\text { Autosomal } \\
\text { dominant SCA }\end{array}$ & Total \\
\hline SCA1 & 1 Salisbury & 1 Sheffield & 2 \\
\hline SCA2 & $\begin{array}{l}5 \text { Belfast (2) } \\
\text { Cambridge (3) }\end{array}$ & 1 Sheffield & 6 \\
\hline SCA3 & 1 Cambridge & 0 & 1 \\
\hline SCA6 & $\begin{array}{l}6 \text { Salisbury (2) } \\
\text { Manchester (3) } \\
\text { Cambridge (1) }\end{array}$ & 1 Sheffield & 7 \\
\hline $\begin{array}{l}\text { DRPLA } \\
\text { Friedreich's }\end{array}$ & 2 Salisbury & 0 & 2 \\
\hline $\begin{array}{l}\text { ataxia } \\
\text { Total cases }\end{array}$ & $\begin{array}{l}4 \text { Sheffield } \\
124\end{array}$ & $\begin{array}{l}0 \\
22\end{array}$ & $\begin{array}{l}4 \\
146\end{array}$ \\
\hline
\end{tabular}

and $\mathrm{SCA} 2-\mathrm{B}^{7}$ and the SCA6 repeats were amplified using primers S-5-F1 and S-5-R1. ${ }^{9}$ Primers Rep1 and Rep2 were used to amplify the SCA1 repeats ${ }^{4}$ and SCA3 repeats were amplified using primers MJD25 and MJD52. ${ }^{5}$ DRPLA expansions were analysed using primers CTG-B37-F and CTG-B37-R ${ }^{19}$ and Friedreich's ataxia genotypes were determined using GAA-104F and GAA-629R. ${ }^{10}$ Five percent DMSO was added to the reaction mixtures for SCA2 and SCA6. PCR products of the SCA and DRPLA reactions were sized on $6 \%$ denaturing polyacrylamide gels compared to a sequence ladder (pGEM $3 \mathrm{Z}(+)$ (Promega)). Homozygotes were amplified twice and all gels were subjected to long exposures to detect any expanded alleles which might have been faint on the autoradiographs.

\section{Results}

To confirm the recently established size ranges of normal alleles in SCA2 and SCA6, we genotyped two populations of Africans, one composed mainly of Nigerians and the other from South Africa. We looked at 272 chromosomes at the SCA2 locus and found one allele larger than the previously determined normal range of $15-29 \mathrm{CAGs}^{6-8}$ but below the putative disease size (fig 1A). In the case of SCA6, we examined 294 chromosomes from the same two African populations and found that all alleles fell into the previously published normal size range for the CAG repeat tract ${ }^{9}$ (fig 1B).

Our clinical sample of 146 cases ascertained from patients referred to molecular genetics laboratories in the UK with SCA-like symptoms was analysed for SCA1, 2, 3, and 6, DRPLA, and FA mutations. Three of 22 cases referred with the diagnosis of autosomal dominant SCA $(14 \%)$ had detectable mutations in addition to 19 of 124 of patients referred with spinocerebellar ataxia where we did not have sufficient family history data available to allow categorisation as familial or sporadic cases $(15 \%)$. The most common SCAs detected in this sample were SCA2 (six cases) and SCA6 (seven cases) (table 1). All expanded mutant alleles fell within previously published disease size ranges. The two cases of DRPLA were not obviously related. One of the cases with DRPLA was from a family which has been described previously. ${ }^{20}$

\section{Discussion}

Identification of causative mutations for SCA1, 2,3 , and 6 allows clinicians to diagnose people definitively by genotyping. Before determining whether patients have these mutations, we first investigated the upper limits of the CAG repeat sizes in normal subjects for SCA2 and SCA6. Since allele frequencies can differ between populations, it is important to test as many populations as possible when establishing normal and disease ranges of CAG repeat tracts. This study tested two African populations, since they are thought to have great allelic diversity. ${ }^{14}$ The presence of an intermediate allele at the SCA2 locus from one Nigerian with 33 repeats could merely indicate a more diverse distribution of non-disease alleles, or it could indicate a disease allele in a patient who has not yet developed the disease. Although we found no alleles outside the putative normal and disease size ranges for SCA6, further studies will be needed to determine the pathological significance of allele sizes that fall between normal and disease ranges. Ideally, the nonpathogenic nature of these long alleles should be confirmed by showing their presence in elderly asymptomatic subjects.

This is the first report of screening for the presence of SCA1, 2, 3, 6, DRPLA, and FA in a large group of patients in the UK referred with clinical features of spinocerebellar ataxia. Fifteen percent of 146 patients had a mutation at one of the six loci tested. The most common causes of SCA in our sample were SCA6 (5\%) and SCA2 (4\%). Interestingly, 3\% (4/124) of isolated cases in our sample were caused by homozygous mutation at the FA locus. Three of these patients presented at age $<25$ years, while the fourth presented at 60 years of age. This confirms the data of Durr et $a l^{11}$ and Filla et $a l,{ }^{10}$ who both found homozygous GAA expansions in the FA gene in subjects with incomplete clinical features of FA presenting after the age of 25 . Although we did not screen for point mutations in this gene, our estimate of the frequency of this disease is not likely to be seriously confounded since GAA expansions are found on $>95 \%$ of disease chromosomes. ${ }^{101113}$

There was some variation in the frequencies and proportions of the various mutations in patients from different centres, which may have been a function of the diagnostic criteria used by referring clinicians or of regional variations in the distribution of these mutations. However, it is likely that the statistical variation owing to the small sample sizes plays a major role in determining this variance.

We found only one case with SCA3 compared to 15 cases with SCA1, 2, and 6 . This contrasts with the data of Silviera et $a l^{11}$ who found that SCA3 accounted for $17 \%$ of SCA cases, and Ranum et $a l^{2}$ who diagnosed SCA3 in $21 \%$ of their SCA patients. More recent studies have suggested that SCA3 may comprise $23-34 \%$ of SCA cases, SCA2 comprises $13-15 \%$ of cases, and SCA1 comprises $6-14 \%$ of cases. ${ }^{23}{ }^{24}$ It is important to note that all of these published studies used populations which were ethnically diverse and that the frequencies of the different SCA mutations may vary in different populations, as is the case with $\mathrm{HD}$ and DRPLA..$^{25}$ All of our patients who had 
identifiable mutations are thought to be English (surnames compatible with being English/European), except for a South African of European origin (SCA1) and a Hong Kong Chinese patient (SCA1).

It is necessary to point out that this study was retrospective with regards to the patients who were referred. Thus, the diagnostic criteria which prompted referral to molecular genetics laboratories were not formalised and may vary from clinician to clinician. The most likely result of this ascertainment procedure is contamination of the patient sample with cases who are more likely to have non-SCA diagnoses. This may affect estimates of the absolute proportions of cases of SCA with specific diagnoses. However, this is unlikely to affect the relative proportions of the different identifiable mutations, that is, the proportion of SCA2 cases versus SCA1 cases.

If the referral criteria of these patients are typical, then clinicians can currently expect molecular diagnoses in about $15 \%$ of their SCA referrals. SCA2 and 6 were the most prevalent in our clinical sample, which emphasises the importance of these recent discoveries.

We are grateful to Trefor Jenkins and Amanda Krause for providing control samples and for critical comments on the manuscript and to C J Hoogendijk for sample collection. This work was supported by the Huntington's Disease Association, UK, and the Rehabilitation and Medical Research Trust (DCR).

1 Genis D, Matilla T, Volpini V, et al. Clinical, neuropathologic and genetic studies of a large spinocerebellar ataxia type 1 (SCA) kindred: (CAG)n expansion and early premonitory signs and symptoms. Neurology 1995;45:24-9.

2 Harding AE. Hereditary ataxias and related disorders. Edinburgh: Churchill Livingstone, 1984.

3 Zoghbi H. The expanding world of ataxins. Nat Genet 1996; 14:237-8

4 Orr HT, Chung M, Banfi S, et al. Expansion of an unstable trinucleotide CAG repeat in spinocerebellar ataxia type 1 . Nat Genet 1993;4:221-6.

5 Kawaguchi Y, Okamoto T, Taniwaki M, et al. CAG expansions in a novel gene for Machado-Joseph disease at chromosome 14q32.1. Nat Genet 1994;8:221-7.

6 Sanpei K, Takano H, Igarashi S, et al. Identification of the spinocerebellar ataxia type 2 gene using a direct identification of repeat expansion and cloning technique, DIRECT. tion of repeat expansion and

7 Pulst SM, Nechiporuk A, Nechiporuk T, et al. Moderate expansion of a normally biallelic trinucleotide repeat in spinocerebellar ataxia type 2 . Nat Genet 1996;14:269-76.

8 Imbert G, Saudou F, Yvert G, et al. Cloning of the gene for spinocerebellar ataxia 2 reveals a locus with high sensitivity to expanded CAG/glutamine repeats. Nat Genet 1994;14: 285-91.
9 Zhuchenko O, Bailey J, Bonnen P, et al. Autosomal dominant cerebellar ataxia (SCA6) associated with small polyglutamine expansions in the $\alpha 1 \mathrm{~A}$-voltage-dependen calcium channel. Nat Genet 1997;15:62-9.

10 Filla A, De Michele G, Cavalcanti F, et al. The relationship between trinucleotide (GAA) repeat length and clinical features in Friedreich ataxia. Am f Hum Genet 1996;59: 554-60.

11 Durr A, Cossee M, Agid Y, et al. Clinical and genetic abnormalities in patients with Friedreich's ataxia. $N$ Engl f Med 1996;335:1169-75.

12 Ross CA. When more is less: pathogenesis of glutamine repeat neurodegenerative diseases. Neuron 1995;15:493-6.

13 Campuzano V, Montermini L, Molto MD, et al. Friedreich's ataxia: autosomal recessive disease caused by an intronic GAA triplet repeat expansion. Science 1996;271:1423-7.

$14 \mathrm{Kidd} \mathrm{KK}$. Can we find genes for schizophrenia? $\mathrm{Am} \mathrm{f} \mathrm{Med}$ Genet (Neuropsychiatric Genet) 1997;74:104-11.

15 Rubinsztein DC, Leggo J, Coles R, et al. Phenotypic characterization of individuals with 30-40 CAG repeats in the Huntington disease (HD) gene reveals HD cases with 36 repeats and apparently normal elderly individuals with repeats and apparently normal elderly individu

16 Rubinsztein DC, Leggo J, Coetzee GA, et al. Sequence variation and size ranges of CAG repeats in the MachadoJoseph disease, spinocerebellar ataxia type 1 and androgen receptor genes. Hum Mol Genet 1995;4:1585-90.

17 Rubinsztein DC, Leggo J, Amos W, et al. Myotonic dystrophy CTG repeats and the associated insertion/deletion polymorphism in human and primate populations. Hum Mol Genet 1994;3:2031-5.

18 Goldman A, Ramsay M, Jenkins T. Absence of myotonic dystrophy in Southern African Negroids is associated with a significantly lower number of CTG trincleotide repeats. $f$ Med Genet 1994;31:37-40.

19 Li SH, McInnis MG, Margolis RL, et al. Novel triplet containing genes in human brain: cloning, expression, and length polymorphisms. Genomics 1993;16:572-9.

20 Connarty M, Dennis NR, Patch C, Macpherson JN, Harvey JF. Molecular re-investigation of patients with Huntington's disease in Wessex reveals a family with dentato-rubra and pallidoluysian atrophy. Hum Genet 1996;97:76-8.

21 Silviera I, Lopes-Cendes I, Kish S, et al. Frequency of spinocerebellar ataxia type 1 , dentatorubropallidoluysian atrophy, and Machado-Joseph disease mutations in a large group of spinocerebellar ataxia patients. Neurology 1996;46: group of

22 Ranum LPW, Lundgren JK, Schut LJ, et al. Spinocerebella ataxia type 1 and Machado-Joseph disease: incidence of CAG expansions among adult-onset ataxia patients from 311 families with dominant, recessive, or sporadic ataxia. Am f Hum Genet 1995;57:603-8.

23 Geschwind DH, Perlman S, Figueroa CP, Treiman LJ, Pulst SM. The prevalence and wide spectrum of the spinocerebellar ataxia type 2 trinucleotide repeat in patients with autosomal dominant cerebellar ataxia. Am $\mathcal{f}$ Hum Genet 1997;60:842-50.

24 Cancel G, Durr A, Didieriean O, et al. Molecular and clinical correlations in spinocerebellar ataxia 2: a study of 32 families. Hum Mol Genet 1997;6:709-15.

25 Rubinsztein DC, Amos W, Leggo J, et al. Mutational bias provides a model for the evolution of Huntington's disease and predicts a general increase in disease prevalence. Nat Genet 1994;7:525-30.

26 Burke JR, Ikeuchi T, Koide R, et al. Dentatorubralpallidoluysian atrophy and Haw River syndrome. Lancet 1994;344:1711-12. 